\title{
Thymol and carvacrol; as antibiotic alternative in green healthy poultry production
}

\author{
Majid Gholami-Ahangaran ${ }^{*}$, Asiye Ahmadi-Dastgerdi², Maryam Karimi-Dehkordi ${ }^{1}$ \\ 1'Department of Clinical Sciences, Veterinary Medicine Faculty, Shahrekord Branch, Islamic Azad University, Shahrekord, IRAN \\ ${ }^{2}$ Department of Food Science and Technology, Ardestan Branch, Islamic Azad University, Ardestan, IRAN
}

\section{*Correspondence to: \\ Dr. Majid Gholami-Ahangaran \\ mgholami6@gmail.com}

Received: 1 May 2020

Accepted: 23 May 2020

ePublished: 16 June 2020

Keywords: Antibacterial, Carvacrol, Thymol, Poultry

\section{Citation:}

Gholami-Ahangaran M, Ahmadi-Dastgerdi A, KarimiDehkordi M. Thymol and carvacrol; as antibiotic alternative in green healthy poultry production. Plant Biotechnol Persa. 2020; 2 (1) :22-25

\section{Abstract}

Thymol and carvacrol as a natural essential oils and phenol compounds, are a component derived from some medicinal plants, such as thyme and oregano species. These compounds have been shown to possess a wide range of biological activities, including antimicrobial, antioxidant, anti-infalmmatory, modulating of immunity respond and anticarcinogenic properties. One of main effects of these compounds that make them as valuable material in poultry medicine is antibacterial effect. The increasing consumption of organic and healthy meat and eggs in human society has made it increasingly necessary to use compounds that are completely natural and do not cause problems for human health. Since antibiotics are chemical compounds that can remain in meat and eggs and cause antibiotic resistance, allergies, mutations and even poisoning in humans. Therefore, utilization of compounds that are natural antibiotics can be easily fed with a higher safety in healthy poultry production. In this regard, the role of thymol and carvacrol as natural antibiotics in the poultry production has been discussed in a recent review.

\section{Introduction}

Today, the use of antibiotics in livestock and poultry for various reasons, such as prevention, treatment of various infectious diseases and to increase the food efficiency is mostly common. Antibiotic residues can lead to various side effects such as carcinogenicity, malformations, mutations, allergies, and drug resistance $[1,2]$. The widespread use of different types of antibiotics, regardless of side effects and withdrawal time, has caused a great deal of concern to consumers of meat and eggs, and there is a growing demand for healthy products [3]. In this regard, phytobiotics can be a good alternative to antibiotics in the breeding of healthy poultry without the mentioned side effects.

Phytochemicals, also referred to as phytobiotics or phytogenics, are natural bioactive compounds that are derived from plants and incorporated into animal feed to enhance productivity. The main bioactive compounds of the phytochemicals are polyphenols, and their composition and concentration vary according to the plant, parts of the plant, geographical origin, harvesting season, environmental factors, storage conditions, and processing techniques [4].

The addition of phytochemicals in the diets alters the intestinal microbiota and reduces microbial toxic metabolites in the gut, owing to their direct antimicrobial properties on various pathogenic bacteria, improving performance [5]. Several plants and their essential oils have been reported to have antibacterial properties for poultry and animal. Carvacrol and thymol are the main compounds that are considered to exhibit antibacterial effect against some important poultry pathogen e.g. Salmonella and E. coli [6-8].

Thymol (C10H14O, 2-isopropyl-5-methylphenol) (Figure 1), as a natural phenol compound, is a component derived from some medicinal plants, such as thyme and oregano species. It has been shown to possess various pharmacological properties including antioxidant, anti-inflammatory, antibacterial, analgesic, antispasmodic, antifungal, antiseptic and antitumor activities. The effects of thymol are largely attributed to its anti-inflammatory, antioxidant and antihyperlipidemic properties [912].

Carvacrol (C10H14O, 2- methyl-5-(1methylethyl) phenol) (Figure 1), is a liquid phenolic which is mostly present in oil of oregano, thyme, pepperwort, savory and wild bergamot. It has been shown to possess a wide range of biological activities, including antibacterial and antifungal, antiviral, modulating of immunity respond, anti-infalmmatory, and anticarcino-

Copyright (C) 2020 The Author(s). This is an open-access article distributed under the terms of the Creative Commons Attribution License (http://creativecommons.org/licenses/by/4.0), which permits unrestricted use, distribution, and reproduction in any medium, provided the original work is properly cited. 
genic properties [13-17].

In this review of literature, we tried to discuss the earlier documents on the effectiveness of thymol and carvacrol on bacterial pathogens in poultry that can be useful in healthy poultry production.

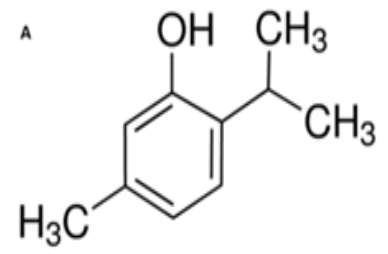

Thymol (2-isopropyl-5-methyiphenol)
Carvacrol (2-methyl-5-(1-methylethyl) phenol)

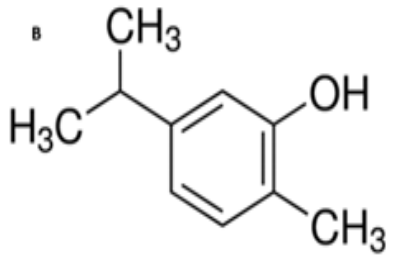

Figure 1. Chemical structures of (A) thymol and (B) carvacrol

\section{Antibacterial effects of thymol and carvacrol}

The antimicrobial activity of essential oils has been extensively investigated in several in vitro studies that showed carvacrol and thymol possess strong antimicrobials effect against pathogenic bacteria such as Escherichia coli, Salmonella typhimurium and Campylobacter spp, which are potential risk factors of enteric infections [18]. Carvacrol and thymol, potent antimicrobial activity, have similar structure, synergistically antibacterial activity [19].

Thymol and carvacrol, represent an alternative to antibiotics in poultry farming, in particular against Clostridium perfringens which is considered the main responsible for necrotic enteritis in chickens. In vitro antibacterial activity of carvacrol and thymol showed strong antibacterial effects against pathogenic strains E. coli, C. perfringens and Salmonella, instead weak activity against beneficial Lactobacillus strains [6]. Marchese et al. (2016) showed E. coli was the most susceptible bacteria to thymol among tested strains (MIC of $187.5 \mu \mathrm{g} / \mathrm{ml}$ ), MBC of $375 \mu \mathrm{g}$ / $\mathrm{ml}$ ), while carvacrol was more active against S. enteritidis (MIC of $187.5 \mu \mathrm{g} / \mathrm{ml}, \mathrm{MBC}$ of $750 \mu \mathrm{g} / \mathrm{ml}$ ) [20].

Carvacrol showed antimicrobial activity against several microbes such as Staphylococcus aureus, Clostridia spp., E. coli, and Salmonella pullorum [21]. Carvacrol and thymol showed higher inhibitory activity against avian E. coli [22].

The essential oils used in poultry diets either individually or in combination or solely pure compounds have shown strong inhibitory effect on Clostridium perfringens and E. coli in the digestive tract and ameliorated intestinal lesions and weight loss than the challenged control birds [23]. Carvacrol, cinnamaldehyde, oregano oil, and thymol were also found to inhibit C. perfringens spore germination and growth in turkey during chilling [7]. Carvacrol, thymol, transcinnamaldehyde, and tetrasodium pyrophosphate on the radio sensitization of E. coli and Salmonella typhi in chicken meat significantly reduced the numbers of these pathogens [24].

Although the antibacterial mechanism of essential oils and their constituents is not fully understood, the mechanism of action of thymol and carvacrol may be related to their hydrophobic property. This property enables the herbal compounds to penetrate into the lipid layers in the bacterial cell wall and mitochondria, rendering them permeable and causing the leakage of cell contents, leading to cell death [25]. Lambert, et al. (2001) exhibited antibacterial effect of thymol and carvacrol against Pseudomonas aeruginosa and Staphylococcus aureus as a result of disruption in membrane integrity, which further affects the $\mathrm{pH}$ homeostasis and balance of inorganic ions [26]. Therefore, antibacterial property of carvacrol and thymol is dependent to their capability to permeabilize, depolarize, and disruption of the cytoplasmic membrane [27]. Cowan (1999) reported that $60 \%$ of essential oil derivatives examined were inhibitory to fungi while $30 \%$ inhibited growth of bacteria [28]. Usually, the essential oils contain high level of phenolic components, including carvacrol, thymol and eugenol have strongest antibacterial properties against food-borne pathogens. Both the phenolics carvacrol and thymol are able to disintegrate the outer membrane of gram-negative bacteria, releasing lipopolysaccharides and increasing the permeability of the cytoplasmic membrane to ATP and depolarize the cytoplasmic membrane [8].

Helander et al. (1998) investigated the antimicrobial mechanism of the two isomeric phenols, carvacrol and thymol, and one phenylpropanoid, cinnamaldehyde, on E. coli and S. typhimurium. It was observed that both carvacrol and thymol disintegrated the outer bacterial membranes in a similar manner, thus intracellular material from cells is transferred to the external medium due to membrane disruption. On the other hand, cinnamaldehyde did not affect the membrane, but showed potent inhibitory antibacterial activity. It was suggested that the phenylpropanoid cinnamadlehyde penetrates the bacterial membrane and thus can reach and affect the inner part of the cell [29]. In agreement to the previous findings, it has also been hypothesized that whole essential oils have greater antibacterial activity than their major components (e.g., solely carvacrol) and this suggests that the minor components in essential oils are also main to the activity and may have a synergistic effect [30]. The two major components of oregano essential oil, carvacrol and thymol, were found to give a synergistic effect when tested against Staphylococcus aureus and Pseudomonas aeruginosa [26]. Synergic effect between carvacrol and its biological precursor p-cymene has been observed. Although, p-cymene is a weak antibacterial substance, it can swell bacterial cell membranes to a larger degree compared to carvacrol. In this way, p-cymene may enable carvacrol to be more easily to transport into the cell so that a synergistic effect is achieved. Furthermore, Zhou et al. (2007) reported that the combination of thymol or carvacrol with EDTA, acetic acid, or citric acid resulted in significantly reduced populations of S. typhimurium. In samples treated with combinations, these antimicrobials exhibited synergistic effects compared with samples treated with thymol, carvacrol, EDTA, acetic acid, or citric acid alone [31]. However, a bacteriostatic or a bactericidal effect of plant extracts, essential oils and herbal compounds is lower than antibiotics [32]. Recently, Moon et al., (2020) showed that that the combination of bacteriophage with thymol and carvacrol significantly reduced Salmonella in chicken meat [9].

Along with the beneficial effects of thymol and carvacrol, it should be noted to possible toxicity of thymol and carvacrol in vital organs. It is difficult to detect the toxicity of essential oils because the toxicity varies based on the compounds and depends on various factors. A study showed that thymol and carvacrol had the most toxic in concentrations of $36-49 \mathrm{mg} / \mathrm{l}$, which are less toxic than some combination of essential oils. There is less risk of accumulation of body tissues [27]. Although, the antibacterial effect was suggested for thymol and carvacrol, but more research must be done on toxicity of them in vivo condition.

However, in most time, the effective levels of thymol and carvacrol are very higher than the cost effective levels in poultry 
production. It should not be overlooked that these compounds can be useful in breeding of organic and healthy chickens that are not allowed to use antibiotics and chemical compounds.

\section{Authors' contribution}

All authors contributed equally to the manuscript.

\section{Conflicts of interest}

The authors declared no competing interests.

\section{Ethical considerations}

Ethical issues (including plagiarism, data fabrication, double publication and etc.) have been completely observed by author.

\section{Funding/Support \\ None.}

\section{References}

1. Dadgarnia M, Gholami-Ahangaran M, Shakerian A. The determination of enrofloxacin residue in quail meat, in Yazd by HPLC. Iran Food Hyg. 2018; 8: 83-90.

2. Teimuri S, Gholami-Ahangaran M, Shakerian A. The comparison of enrofloxacin residue in chicken and turkey meat, by high performance liquid chromatography in Isfahan province. Iran Food Hyg. 2018; 8: 95-100.

3. Gholami-Ahangaran M, Ghasemi Pirbalouti A, Farasat M, Fasihi K. Antimicrobial effect of Zataria multiflora, Thymus daenensis, Althea officinalis, and Urtica dioica on growth of Escherichia coli isolated from poultry colibacillosis. Iran J Vet Mic. 2015; 1: 1-10.

4. Alagawany M, El-Hack MEA, Farag MR, Tiwari R, Dhama $\mathrm{K}$. Biological effects and modes of action of carvacrol in animal and poultry production and health - a review. Adv Anim Vet Sci. 2015; 3(2): 73-84.

5. Dehghani-Ashkezari A, Gholami-Ahangaran M, Fathi-Hafshejani E. The use of garlic extract in reducing the side effects of Eimeria tenella on the growth indices and mucosal tissue of the cecum in broilers. Iran Anim Biol. 2019; 11(2): 45-53.

6. Du E, Gan L, Li Z, Wang W, Liu D, Guo Y. In vitro antibacterial activity of thymol and carvacrol and their effects on broiler chickens challenged with Clostridium perfringens. J Anim sci Biotechnol. 2015; 6: 58.

7. Juneja VK, Friedman M. Carvacrol, cinnamaldehyde, oregano oil, and thymol inhibit Clostridium perfringens spore germination and outgrowth in ground Turkey during chilling. J Food Prot. 2007; 70: 218-222.

8. Xu J, Zhou F, Ji BP, Pei RS, Xu N. Carvacrol and thymol had desired antimicrobial effect on E. coli. The antibacterial effects were attributed to their ability to permeabilize and depolarize the cytoplasmic membrane. Lett Appl Microbiol. 2008; 47: 174-179.

9. Moon SH, Waite-Cusic J, Huang E. Control of Salmonella in chicken meat using a combination of a commercial bacteriophage and plant-based essential oils. Food Control. 2019; https://doi.org/10.1016/j.foodcont.2019.106984

10. El-Hack ME, Alagawany M, Farag MR, Tiwari R, Karthik K, Dhama K, Zorriehzahra J, Adel M. Beneficial impacts of thymol essential oil on health and production of animals, fish and poultry: a review. J Essent Oil Res. 2016; DOI: 10.1080/10412905.2016.1153002

11. Bahmani M, Saki K, Rafieian-Kopaei M. Medicinal Plants of
Thyme land in Iran. Saarbrücken: Lambert Academic Publishing; 2015.

12. Guerra-Boone L, Alvarez-Roman R, Salazar-Aranda R, Torres-Cirio A, Rivas-Galindo VM, de Torres VM, Gonzalez G, Perez-Lopez LA. Antimicrobial and antioxidant activities and chemical characterization of essential oils of Thymus vulgaris, Rosmarinus officinalis, and Origanum majorana from northeastern Mexico. Pak J Pharm Sci. 2015; 28: 363-369.

13. Khazdair MR, Ghorani V, Alavinezhad A, Boskabady MH. Pharmacological effects of Zataria multiflora Boiss L. and its constituents focus on their anti-inflammatory, antioxidant, and immunomodulatory effects. Fundam Clin Pharmacol. 2018; 32(1): 26-50.

14. Fitsiou E, Anestopoulos I, Chlichlia K, Galanis A, Kourkoutas I, Panayiotidis MI, Pappa A. Antioxidant and antiproliferative properties of the essential oils of Satureja thymbra and Satureja parnassica and their major constituents. Anticancer Res. 2016; 36(11): 5757-5763.

15. Sánchez C, Aznar R, Sánchez G. The effect of carvacrol on enteric viruses. Int J Food Microbiol. 2015; 192: 72-76.

16. Özkan A, Erdogan A. A comparative evaluation of antioxidant and anticancer activity of essential oil from Origanum onites (Lamiaceae) and its two major phenolic components. Turkish J Biol. 2011; 35(6): 735-742.

17. Sharifi-Rad M, Varoni EM, Iriti M, Martorell M, Setzer WN, del Mar Contreras M, Salehi B, Soltani-Nejad A, Rajabi S, Tajbakhsh M, Sharifi-Rad J. Carvacrol and human health: A comprehensive review. Phytother Res. 2018; 32(9): 16751687.

18. Hippenstiel F, Abdel-Wareth AAA, Kehraus S, Sudekum $\mathrm{KH}$. Effects of selected herbs and essential oils, and their active components on feed intake and performance of broilers e a review. Arch Geflügelk. 2011; 75: 226-234.

19. Bassole IH, Juliani HR. Essential oils in combination and their antimicrobial properties. Molecules. 2012; 17: 39894006.

20. Marchese A, Orhan IE, Daglia M, Barbieri R, Lorenzo AD, Nabavi SF, Gortzi O, Izadi M, Nabavi SM. Antibacterial and antifungal activities of thymol: a brief review of the literature. Food Chem. 2016; doi: http://dx.doi.org/10.1016/j. foodchem.2016.04.111

21. Dahiya JP, Wilkie DC, Van Kessel AG, Drew MD. Potential strategies for controlling necrotic enteritis in broiler chickens in post-antibiotic era. Anim Feed Sci Technol. 2006; 129: 60-88.

22. Penalver P, Huerta B, Borge C, Astorga R, Romero R, Perea A. Antimicrobial activity of five essential oils against origin strains of the Enterobacteriaceae family. APMIS. 2005; 113 : 1-6.

23. Jerzsele A, Szeker K, Csizinszky R, Gere E, Jakab C, Mallo JJ, Galfi P. Efficacy of protected sodium butyrate, a protected blend of essential oils, their combination, and Bacillus amyloliquefaciens spore suspension against artificially induced necrotic enteritis in broilers. Poultry Sci. 2012; 91: 837-843.

24. Lacroix M, Chiasson F. The influence of MAP condition and active compounds on the radiosensitization of Escherichia coli and Salmonella typhi present in chicken breast. Radiat Phys Chem. 2004; 71: 69-72.

25. Burt S. Essential oils: their antibacterial properties and potential applications in foods e a review. Int J Food Microbiol. 2004; 94: 223-253. 
26. Lambert RJW, Skandamis PN, Coote PJ, Nychas GJE. A study of the mimum inhibitory concentration and mode of action of oregano essential oil, thymol and carvacrol. J Appl Microbiol. 2001; 91: 453-462.

27. Memar MY, Raei P, Alizadeh N, Aghdam M A, Kafil HS. Carvacrol and thymol: strong antimicrobial agents against resistant isolates. Rev Med Microbiol. 2017; 28(2): 63-68.

28. Cowan MM. Plant products as antimicrobial agents. Clin Microbiol Rev. 1999; 12: 564-582.

29. Helander M, Alakomi H, Latva-Kala K, Mattila-Sandholm T, Pol I, Smid EJ, Gorris LGM, Wright AV. Characterization of the action of selected essential oil components on Gram-negative bacteria. J Agric Food Chem. 1998; 46: 35903595.

30. Brenes A, Roura E. Essential oils in poultry nutrition: main effects and modes of action. Anim Feed Sci Technol. 2010; 158: 1-14.

31. Florou-Paneri P, Palatos G, Govaris A, Botsoglou D, Giannenas I, Ambrosiadis I. Oregano herb versus oregano essential oil as feed supplements to increase the oxidative stability of Turkey meat. Int J Poult Sci. 2005; 4: 866-871.

32. Zhu X, Zhang H, Lo R. Phenolic compounds from the leaf extract of artichoke (leaf extract of artichoke (Cynara scolymus L.) and their antimicrobial activities. J Agricul Food Chem. 2004; 52: 7272-7278. 